\title{
Analysis of XFEL serial diffraction data from individual crystalline fibrils
}

\author{
David H. Wojtas,${ }^{a}$ Kartik Ayyer,${ }^{b}$ Mengning Liang,${ }^{c}$ Estelle \\ Mossou,${ }^{d, i}$ Filippo Romoli,${ }^{k}$ Carolin Seuring,${ }^{b}$ Kenneth R. Beyerlein,${ }^{b}$ \\ Richard J. Bean,$g$ Andrew J. Morgan,${ }^{b}$ Dominik Oberthuer,${ }^{b}$ Holger \\ Fleckenstein,${ }^{b}$ Michael Heymann,${ }^{b, h}$ Cornelius Gati,${ }^{b}$ Oleksandr \\ Yefanov,${ }^{b}$ Miriam Barthelmess,${ }^{b}$ Eirini Ornithopoulou,$j$ Lorenzo \\ Galli,${ }^{b}$ P. Lourdu Xavier,${ }^{b, m}$ W. L. Ling,${ }^{n}$ Matthias Frank,${ }^{l}$ Chun \\ Hong Yoon , ${ }^{c}$ Thomas A. White,${ }^{b}$ Saša Bajt,${ }^{p}$ Anna Mitraki,$j$ \\ Sebastien Boutet,${ }^{c}$ Andrew Aquila,${ }^{c}$ Anton Barty,${ }^{b}$ V. Trevor \\ Forsyth ${ }^{i, d}$ Henry N. Chapman ${ }^{b, e, f}$ And Rick P. Millane $a *$ \\ ${ }^{a}$ Department of Electrical and Computer Engineering, University of Canterbury, \\ Christchurch, New Zealand, ${ }^{b}$ Center for Free-Electron Laser Science, DESY, \\ Hamburg, Germany, ${ }^{c}$ Linac Coherent Light Source, SLAC National Accelerator \\ Laboratory, Menlo Park, California, USA, ${ }^{d}$ Institut Laue-Langevin, Grenoble, \\ France, ${ }^{e}$ Department of Physics, University of Hamburg, Hamburg, Germany, \\ ${ }^{f}$ Centre for Ultrafast Imaging, University of Hamburg, Hamburg, Germany, \\ ${ }^{g}$ European XFEL GmbH, Hamburg, Germany, ${ }^{h}$ Max Planck Institute of \\ Biochemistry, Martinsried, Germany, ${ }^{i}$ Faculty of Natural Sciences, Keele University, \\ UK, ${ }^{j}$ Department of Materials Science and Technology, University of Crete and \\ IESL/FORTH, Crete, Greece, ${ }^{k}$ European Synchrotron Radiation Facility, Grenoble, \\ France, ${ }^{l}$ Lawrence Livermore National Laboratory, Livermore, California, USA, \\ ${ }^{m}$ Max-Planck Institute for the Structure and Dynamics of Matter, Hamburg,
}


Germany, ${ }^{n}$ Université Grenoble Alpes, Grenoble, France, and ${ }^{p}$ Photon Science, DESY, Hamburg, Germany.E-mail: rick.millane@canterbury.ac.nz 


\section{Supplementary information}

Table 1. Measured and predicted $R$ values.

\begin{tabular}{|c|c|c|c|c|c|c|c|}
\hline$(h, k)$ & $R_{h k}\left(\AA^{-1}\right)$ & $R_{i}^{o b s}\left(\AA^{-1}\right)$ & $\Delta R\left(\AA^{-1}\right)$ & Intensity & $R_{\mathrm{i}}\left(\AA^{-1}\right)$ & $\Delta R\left(\AA^{-1}\right)$ & Intensity \\
\hline$(0,1)$ & 0.0385 & 0.0387 & 0.0002 & $?$ & & & \\
\hline$(1,0)$ & 0.0559 & 0.0557 & -0.0002 & $\mathrm{~m}$ & 00589 & 0.003 & YS \\
\hline$(-1,1)$ & 0.0604 & 0.0605 & 0.0001 & $\mathrm{~m}$ & 0.0589 & -0.0015 & vs \\
\hline$(1,1)$ & 0.0745 & 00764 & 0.0019 & $\mathrm{w}$ & 00743 & -0.0002 & w \\
\hline$(0,2)$ & 0.077 & 0.0604 & -0.0006 & $\mathrm{~W}$ & 0.0145 & -0.0027 & W \\
\hline$(-1,2)$ & 0.0845 & 0.0843 & -0.0002 & $\mathrm{~s}$ & 0.0843 & -0.0002 & $\mathrm{~m}$ \\
\hline$(1,2)$ & 0.1047 & & & & & & \\
\hline$(-2,1)$ & 0.1098 & 1098 & 0.0000 & & 01103 & 0.0005 & \\
\hline$(2,0)$ & 0.1117 & 0.1098 & -0.0019 & $\mathrm{~S}$ & 0.1103 & -0.0014 & $\mathrm{~m}$ \\
\hline$(0,3)$ & 0.1155 & & & & 01156 & 0.0001 & \\
\hline$(-1,3)$ & 0.1166 & & & & 0.1156 & -0.001 & $\mathrm{w}$ \\
\hline$(-2,2)$ & 0.1208 & 0.1207 & -0.0001 & $\mathrm{w}$ & & & \\
\hline$(2,1)$ & 0.126 & & & & & & \\
\hline$(1,3)$ & 0.139 & & & & 01416 & 0.0026 & \\
\hline$(-2,3)$ & 0.1417 & & & & 0.1416 & -0.0001 & $\mathrm{w}$ \\
\hline$(2,2)$ & 0.1491 & 01512 & 0.0021 & $\mathrm{~m}$ & & 0.0026 & \\
\hline$(-1,4)$ & 0.1517 & 0.1512 & -0.0005 & $\mathrm{~m}$ & 0.1517 & 0 & $\mathrm{~m}$ \\
\hline$(0,4)$ & 0.1539 & & & & & -0.0022 & \\
\hline$(-3,1)$ & 0.1634 & & & & & & \\
\hline$(3,0)$ & 0.1676 & & 0.0006 & & & 0 & \\
\hline$(-3,2)$ & 0.1682 & 0.1682 & 0.0000 & $\mathrm{~s}$ & 0.1676 & -0.0006 & $\mathrm{w}$ \\
\hline$(-2,4)$ & 0.1689 & & -0.0007 & & & -0.0013 & \\
\hline$(1,4)$ & 0.175 & & & & & & \\
\hline$(2,3)$ & 0.1776 & & 0.0006 & & & & \\
\hline$(3,1)$ & 0.1801 & 0.1782 & -0.0019 & vw & & & \\
\hline$(-3,3)$ & 0.1812 & & -0.0030 & & & & \\
\hline$(-1,5)$ & 0.1881 & & & & & & \\
\hline$(0,5)$ & 0.1924 & & & & & & \\
\hline$(-2,5)$ & 0.1993 & & 0.0003 & & & -0.0004 & \\
\hline$(3,2)$ & 0.1999 & 0.2 & 0.0001 & $\mathrm{~s}$ & 0.1989 & -0.001 & vw \\
\hline$(-3,4)$ & 0.2008 & & -0.0007 & & & -0.0019 & \\
\hline$(2,4)$ & 0.2093 & & & & & & \\
\hline$(1,5)$ & 0.2119 & & & & & & \\
\hline$(-4,1)$ & 0.2181 & & & & & & \\
\hline$(-4,2)$ & 0.2196 & & & & & & \\
\hline$(4,0)$ & 0.2234 & & & & & & \\
\hline$(3,3)$ & 0.2236 & & & & & & \\
\hline$(-3,5)$ & 0.2254 & & & & & & \\
\hline$(-4,3)$ & 0.2276 & & & & & & \\
\hline$(4,1)$ & 0.235 & & & & & & \\
\hline $\begin{array}{c}(-4,4) \\
(2,5)\end{array}$ & $\begin{array}{c}0.2416 \\
0.243\end{array}$ & & & & & & \\
\hline$\frac{(2,0)}{(3,4)}$ & $\frac{0.243}{0.2515}$ & & & & & & \\
\hline$(4,2)$ & 0.252 & & & & & & \\
\hline$(-4,5)$ & 0.2605 & 0.261 & 0.0005 & $\mathrm{vw}$ & & & \\
\hline$(-5,2)$ & 0.2727 & & & & & & \\
\hline$(-5,1)$ & 0.2733 & & & & & & \\
\hline$(4,3)$ & 0.2733 & & & & & & \\
\hline$(-5,3)$ & 0.2775 & 0.2774 & -0.0001 & $\mathrm{w}$ & & & \\
\hline$(5,0)$ & 0.2793 & 0.2174 & -0.0019 & $\mathrm{~W}$ & & & \\
\hline$(3,5)$ & 0.2818 & & & & & & \\
\hline$(-5,4)$ & 0.2874 & 02875 & 0.0001 & $\mathrm{~m}$ & & & \\
\hline$(5,1)$ & 0.2903 & & -0.0028 & $\mathrm{~m}$ & & & \\
\hline
\end{tabular}

$R_{h k}$ are values for the derived unit cell and $R_{i}^{\text {obs }}$ are the measured values. $\Delta R=R_{i}^{\text {obs }}-R_{h k}$. Peak intensities are denoted: $\mathrm{vw}=$ very weak, $\mathrm{w}=$ weak, $\mathrm{m}=$ medium, $\mathrm{s}=$ strong, $\mathrm{vs}=$ very strong. 
Table 2. Measured structure amplitudes $\left|F_{h k l}\right|$.

\begin{tabular}{|c|c|c|c|}
\hline$(h, k)$ & $R_{h k}\left(\AA^{-1}\right)$ & $F_{h k 0}$ & $F_{h k 1}$ \\
\hline$(0,0)$ & 0.0 & $\mathrm{U}$ & $\mathrm{T}$ \\
\hline$(0,1)$ & 0.0384 & $\mathrm{U}$ & $\mathrm{T}$ \\
\hline$(0,2)$ & 0.0769 & 38 & $\mathrm{~T}$ \\
\hline$(0,3)$ & 0.1153 & 64 & $\mathrm{~T}$ \\
\hline$(0,4)$ & 0.1537 & 51 & $\mathrm{~T}$ \\
\hline$(0,5)$ & 0.1922 & 24 & 33 \\
\hline$(0,6)$ & 0.2306 & $\mathrm{~T}$ & - \\
\hline$(0,7)$ & 0.2691 & $\mathrm{~T}$ & - \\
\hline$(1,-7)$ & 0.2626 & $\mathrm{~T}$ & - \\
\hline$(1,-6)$ & 0.2251 & $\mathrm{~T}$ & - \\
\hline$(1,-5)$ & 0.1881 & 18 & 06 \\
\hline$(1,-4)$ & 0.1517 & 54 & 19 \\
\hline$(1,-3)$ & 0.1166 & 44 & $\mathrm{~T}$ \\
\hline$(1,-2)$ & 0.0846 & 65 & 25 \\
\hline$(1,-1)$ & 0.0604 & $\mathrm{U}$ & $\overline{\mathrm{U}}$ \\
\hline$(1,0)$ & 0.0558 & $\mathrm{U}$ & 54 \\
\hline$(1,1)$ & 0.0743 & 14 & 06 \\
\hline$(1,2)$ & 0.1044 & 61 & 13 \\
\hline$(1,3)$ & 0.1386 & $\mathrm{~T}$ & $\mathrm{~T}$ \\
\hline$(1,4)$ & 0.1746 & 74 & $\mathrm{~T}$ \\
\hline$(1,5)$ & 0.2115 & 18 & $\mathrm{~T}$ \\
\hline$(1,6)$ & 0.2488 & 14 & \\
\hline$(1,7)$ & 0.2865 & 20 & \\
\hline$(2,-7)$ & 0.2678 & $\mathrm{~T}$ & \\
\hline$(2,-6)$ & 0.2332 & $\mathrm{~T}$ & \\
\hline$(2,-5)$ & 0.2 & 65 & $\mathrm{~T}$ \\
\hline$(2,-4)$ & 0.1691 & 17 & 01 \\
\hline$(2,-3)$ & 0.1419 & 03 & 18 \\
\hline$(2,-2)$ & 0.1209 & 12 & $\mathrm{~T}$ \\
\hline$(2,-1)$ & 0.1098 & 55 & $\bar{T}$ \\
\hline$(2,0)$ & 0.1116 & 68 & $\mathrm{~T}$ \\
\hline$(2,1)$ & 0.1257 & 22 & 12 \\
\hline$(2,2)$ & 0.1487 & 25 & 09 \\
\hline$(2,3)$ & 0.1771 & 53 & $\mathrm{~T}$ \\
\hline$(2,4)$ & 0.2087 & 21 & $\bar{T}$ \\
\hline$(2,5)$ & 0.2424 & $\mathrm{~T}$ & \\
\hline$(2,6)$ & 0.2772 & 07 & \\
\hline$(3,-7)$ & 0.2841 & $\mathrm{~T}$ & \\
\hline$(3,-6)$ & 0.2537 & $\mathrm{~T}$ & \\
\hline$(3,-5)$ & 0.2256 & $\mathrm{~T}$ & \\
\hline$(3,-4)$ & 0.201 & 38 & $\mathrm{~T}$ \\
\hline$(3,-3)$ & 0.1813 & 16 & $\bar{T}$ \\
\hline$(3,-2)$ & 0.1682 & 60 & $\mathrm{~T}$ \\
\hline$(3,-1)$ & 0.1633 & 07 & $\mathrm{~T}$ \\
\hline$(3,0)$ & 0.1673 & 100 & $\overline{\mathrm{T}}$ \\
\hline$(3,1)$ & 0.1797 & 16 & $\mathrm{~T}$ \\
\hline$(3,2)$ & 0.1988 & 48 & $\mathrm{~T}$ \\
\hline$(3,3)$ & 0.223 & $\mathrm{~T}$ & \\
\hline$(3,4)$ & 0.2508 & 14 & \\
\hline$(3,5)$ & 0.281 & $\mathrm{~T}$ & \\
\hline$(4,-6)$ & 0.2837 & $\mathrm{~T}$ & \\
\hline$(4,-5)$ & 0.2608 & $\mathrm{~T}$ & \\
\hline$(4,-4)$ & 0.2418 & $\mathrm{~T}$ & \\
\hline$(4,-3)$ & 0.2277 & $\mathrm{~T}$ & \\
\hline$(4,-2)$ & 0.2196 & $\bar{T}$ & \\
\hline$(4,-1)$ & 0.218 & $\mathrm{~T}$ & \\
\hline$(4,0)$ & 0.2231 & 02 & \\
\hline$(4,1)$ & 0.2345 & 06 & \\
\hline$(4,2)$ & 0.2514 & 08 & \\
\hline$(4,3)$ & 0.2726 & $\mathrm{~T}$ & \\
\hline$(4,4)$ & 0.2973 & $\mathrm{~T}$ & \\
\hline$(5,-4)$ & 0.2876 & 31 & \\
\hline$(5,-3)$ & 0.2776 & $\mathrm{~T}$ & \\
\hline$(5,-2)$ & 0.2727 & $\mathrm{~T}$ & \\
\hline$(5,-1)$ & 0.2731 & 15 & \\
\hline$(5,0)$ & 0.2789 & $\mathrm{~T}$ & \\
\hline$(5,1)$ & 0.2897 & 24 & \\
\hline
\end{tabular}

Unmeasured and below threshold values are denoted $\mathrm{U}$ and $\mathrm{T}$, respectively. For $l=1$, the values $\left|F_{h k 1}\right|$ represent $\sqrt{\left|F_{h k 1}\right|^{2}+\left|F_{\bar{h} \bar{k} 1}\right|^{2}}$, as described in the text. 